\title{
ON THE ORBITS OF ANALYTIC FUNCTIONS WITH RESPECT TO A POMMIEZ TYPE OPERATOR
}

\author{
O.A. IVANOVA, S.N. MELIKHOV \\ Dedicated to the memory of Professor \\ Igor' Fedorovich Krasichkov-Ternovskii
}

\begin{abstract}
Let $\Omega$ be a simply connected domain in the complex plane containing the origin, $A(\Omega)$ be the Fréchet space of all functions analytic in $\Omega$. A function $g_{0}$ analytic in $\Omega$ such that $g_{0}(0)=1$ defines the Pommiez type operator which acts continuously and linearly in $A(\Omega)$. In this article we describe cyclic elements of the Pommiez type operator in space $A(\Omega)$. Similar results were obtained early for functions $g_{0}$ having no zeroes in domain $\Omega$.
\end{abstract}

Keywords: Pommiez operator, cyclic element, analytic function.

Mathematics Subject Classification: 47A16, 47B38, 46E10

Let $\Omega$ be a simply connected domain in $\mathbb{C}$ containing the origin; $A(\Omega)$ be the Fréchet space of all functions analytic in $\Omega$. Let a function $g_{0} \in A(\Omega)$ be such that $g_{0}(0)=1$. We introduce a Pommiez type operator as follows. For $f \in A(\Omega)$ we denote

$$
D_{0, g_{0}}(f)(t):= \begin{cases}\frac{f(t)-g_{0}(t) f(0)}{t}, & t \neq 0, \\ f^{\prime}(0)-g_{0}^{\prime}(0) f(0), & t=0 .\end{cases}
$$

Operator $D_{0, g_{0}}$ maps linearly and continuously $A(\Omega)$ into itself. For $g_{0} \equiv 1$ we let $D_{0}:=D_{0, g_{0}}$. Operator $D_{0}$ is called Pommiez operator after the works by M. Pommiez [10]-[13], where successive error terms of Taylor series were studied for the functions analytic in the unit circle.

In the present work we deal with cyclic elements of operator $D_{0, g_{0}}$ in $A(\Omega)$. Given a locally convex space $E$ and a linear continuous operator $A: E \rightarrow E$, an element $x \in E$ is called a cyclic element of operator $A$ if the system (orbit of $x)\left\{A^{n}(x): n \geqslant 0\right\}$ is complete in $E$, i.e., the closure of its linear span in $E$ coincides with $E$.

For $g_{0} \equiv 1$, the completeness conditions for the system $\left\{D_{0, g_{0}}^{n}(f): n \geqslant 0\right\}$ in $A(\Omega)$ were obtained by M.G. Khaplanov [5] and N.I. Nagnibida [4] (in the case $\Omega$ is a circle). In works by Y.A. Kaz'min [2] and N.E. Linchuk [3] (also for $g_{0} \equiv 1$ ) there were obtained cyclicity critetions of $f \in A(\Omega)$ w.r.t. operator $D_{0, g_{0}}$ for an arbitrary simply connected domain $\Omega$ and a finite connected domain $G$ in $\overline{\mathbb{C}}$ containing the origin. In work [9], Yu.S. Linchuk proved necessary and sufficient conditions for the completeness in $A(\Omega)$ of the system $\left\{A^{n}(f): n \geqslant 0\right\}$, where $A(g)(z)=D_{0}(g)(z)+g(0) \varphi(z), g \in A(\Omega)$, and $\varphi \in A(\Omega)$ is a fixed function [9, Corollary]. At that, it was assumed in [9] in the corresponding criterion that function $1-z \varphi(z)$ does not vanish in $\Omega$. It is easy to see that $A=D_{0, g_{0}}$, where $g_{0}(z)=1-z \varphi(z)$.

In the present paper we prove a cyclicity criterion for a function $f \in A(\Omega)$ w.r.t. operator $D_{0, g_{0}}$ for an arbitrary function $g_{0} \in A(\Omega)$ (such that $g_{0}(0)=1$ ) not necessary non-zero in the

O.A. Ivanova, S.N. Melikhov, On the orbits of Analytic functions with respect to a Pommiez TYPE OPERATOR.

(c) Ivanova O.A., Melikhov S.N. 2015.

Submitted May 14, 2015. 
whole domain $\Omega$. In [9], by means [9, Lm.], see Lemma 1 in what follows, the proof of the corresponding criterion is reduced to the case $g_{0} \equiv 1$ studied in [3, Thm. 2] by means of the theory of characteristic functions for linear continuous operators in $A(\Omega)$ [8]. Such reduction is impossible if $g_{0}$ has zeroes in $\Omega$.

In the present work we employ Köthe-Silva-Grothendieck duality between $A(\Omega)$ and the space $A_{0}(\overline{\mathbb{C}} \backslash \Omega)$ of germs of functions analytic in $\overline{\mathbb{C}} \backslash \Omega$ and vanishing at infinity [8] as well as the results on limit values for Cauchy type integrals [1, Ch. I, Sect. 4].

We fix a function $g_{0} \in A(\Omega)$ such that $g_{0}(0)=1$. Following [6], [7], for $z \in \Omega$ we introduce $a$ shift operator for $D_{0, g_{0}}$

$$
T_{z}(f)(t):= \begin{cases}\frac{t f(t) g_{0}(z)-z f(z) g_{0}(t)}{t-z}, & t \neq z, \\ z g_{0}(z) f^{\prime}(z)-z f(z) g_{0}^{\prime}(z)+f(z) g_{0}(z), & t=z\end{cases}
$$

$f \in A(\Omega)$. For each $z \in \Omega$ operator $T_{z}$ maps linearly and continuously $A(\Omega)$ into itself.

We shall make use of the following abstract functional cyclicity criterion [9, Lm.]:

Lemma 1. The following statements are equivalent:

(i) $f$ is a cyclic element of operator $D_{0, g_{0}}$ in $A(\Omega)$.

(ii) System $\left\{T_{z}(f): z \in \Omega\right\}$ is complete in $A(\Omega)$.

This result was proven in [9] without assumption that $g_{0}$ has zeroes in $\Omega$.

In what follows by $A(\Omega)^{\prime}$ we denote the topologically dual space for $A(\Omega)$.

Theorem 1. Let $\Omega$ be a simply connected domain in $\mathbb{C}, 0 \in \Omega$. The following statements are equivalent

(i) $f$ is not a cyclic element of operator $D_{0, g_{0}}$ in $A(\Omega)$.

(ii) Functions $f$ and $g_{0}$ have common zeroes in $\Omega$ or there exists a rational function $R$ such that $f=g_{0} R$.

Proof. $(i i) \Rightarrow(i)$ : Suppose that $f$ and $g_{0}$ have a common zero $\alpha \in \Omega$. Then $D_{0, g_{0}}^{n}(f)(\alpha)=0$ for each $n \geqslant 0$ and each function in $F:=\operatorname{span}\left\{D_{0, g_{0}}^{n}(f): n \geqslant 0\right\}$ vanishes at $\alpha$ and the same is true for each function in $\bar{F}$, which is the closure of $F$ in $A(\Omega)$. Since there exists a function in $A(\Omega)$ not vanishing at $\alpha$, then $f$ is not a cyclic element of $D_{0, g_{0}}$ in $A(\Omega)$.

Suppose that $f=g_{0} R$, where $R$ is a rational function. Let $R=P / Q$, where $P, Q$ are polynomials, $Q \not \equiv 0$. Let us construct a functional $\varphi \in A(\Omega)^{\prime} \backslash\{0\}$ such that $\varphi\left(T_{z}(f)\right)=0$ for each $z \in \Omega$. In order to do it, we construct a function $\gamma \neq \equiv 0$ analytic in $\mathbb{C} \backslash\{0\}$ such that $\gamma(\infty)=0$ and

$$
\frac{1}{2 \pi i} \int_{|t|=r} \gamma(t) T_{z}(f)(t) d t=0, \quad z \in \Omega .
$$

Here $r>0$ is such that the circle $\{t \in \mathbb{C}:|t| \leqslant r\}$ is contained in $\Omega$ and polynomial $Q$ does not vanish on the circle $|t|=r$. Let $\gamma(t):=\sum_{k=0}^{N} \frac{c_{k}}{t^{k+1}} ;$ numbers $c_{k}$ and $N \in \mathbb{N}$ will be determined later.

Condition (1) is equivalent to

$$
\frac{1}{2 \pi i} \int_{|t|=r} \frac{\gamma(t) g_{0}(t)}{Q(t)} \frac{t P(t) Q(z)-z P(z) Q(t)}{t-z} d t=0, \quad z \in \Omega .
$$


If $|z|=r$, then integrand is defined naturally at $t=z$. Since $P$ and $Q$ are polynomials, there exist $M \in \mathbb{N}$ and polynomials $b_{j}, 0 \leqslant j \leqslant M$, such that

$$
\frac{t P(t) Q(z)-z P(z) Q(t)}{t-z}=\sum_{j=0}^{M} b_{j}(z) t^{j} .
$$

The identity (2) can be rewritten as

$$
\frac{1}{2 \pi i} \int_{|t|=r} \gamma(t) \frac{g_{0}(t)}{Q(t)} \sum_{j=0}^{M} b_{j}(z) t^{j} d t=0, \quad z \in \Omega,
$$

i.e.,

$$
\sum_{j=0}^{M}\left(\sum_{k=0}^{N} c_{k} \frac{1}{2 \pi i} \int_{|t|=r} \frac{g_{0}(t)}{Q(t)} t^{j-k-1} d t\right) b_{j}(z)=0, \quad z \in \Omega .
$$

These identities hold true if

$$
\sum_{k=0}^{N} c_{k} a_{j k}=0,0 \leqslant j \leqslant M
$$

where

$$
a_{j k}:=\frac{1}{2 \pi i} \int_{|t|=r} \frac{g_{0}(t)}{Q(t)} t^{j-k-1} d t .
$$

We fix $N>M$. Then system (3) has a nontrivial solution $c_{k}, 0 \leqslant k \leqslant M$. Hence, for a non-zero functional $\varphi \in A(\Omega)^{\prime}$ such that

$$
\varphi(f):=\frac{1}{2 \pi i} \int_{|t|=r} \gamma(t) f(t) d t, \quad f \in A(\Omega),
$$

the identities

$$
\varphi\left(T_{z}(f)\right)=0, \quad z \in \Omega,
$$

hold true. Thus, the system $\left\{T_{z}(f): z \in \Omega\right\}$ is incomplete in $A(\Omega)$ and by Lemma $1, f$ is not a cyclic element of operator $D_{0, g_{0}}$ in $A(\Omega)$.

$(i) \Rightarrow(i i)$ : Suppose that $f$ is not a cyclic element of operator $D_{0, g_{0}}$ in $A(\Omega)$. Then by [8] there exist a function $\gamma \in A_{0}(\overline{\mathbb{C}} \backslash \Omega), \gamma \neq \equiv 0$, a closed rectifiable Jordan curve $\Gamma$ lying in the analyticity domain of $\gamma$ and in $\Omega$, such that

$$
\frac{1}{2 \pi i} \int_{\Gamma} \gamma(t) \frac{t f(t) g_{0}(z)-z f(z) g_{0}(t)}{t-z} d t=0, z \in \operatorname{int} \Gamma .
$$

Here $A_{0}(\overline{\mathbb{C}} \backslash \Omega)$ denotes the space of analytic functions in $\overline{\mathbb{C}} \backslash \Omega$ vanishing at infinity; int $\Gamma$ is the interior of curve $\Gamma$.

Let $f$ and $g_{0}$ have no common zeroes in $\Omega$. We denote

$$
u(z):=\frac{1}{2 \pi i} \int_{\Gamma} \frac{t \gamma(t) f(t)}{t-z} d t, \quad v(z):=\frac{1}{2 \pi i} \int_{\Gamma} \frac{\gamma(t) g_{0}(t)}{t-z} d t, \quad z \in \operatorname{int} \Gamma .
$$

Functions $u$ and $v$ can be analytically continued in $\Omega$. It follows from (4) that

$$
g_{0}(z) u(z)-z f(z) v(z)=0, \quad z \in \Omega .
$$

Since $f$ and $g_{0}$ have no common zeroes in $\Omega$, then

$$
v_{0}:=\frac{v}{g_{0}} \in A(\Omega) \quad \text { and } \quad u_{0}:=\frac{u}{f} \in A(\Omega) .
$$


At that, $u_{0}(z)=z v_{0}(z), z \in \Omega$. By the Cauchy integral formula

$$
\frac{1}{2 \pi i} \int_{\Gamma} \frac{t f(t) v_{0}(t)}{t-z} d t=f(z) u_{0}(z), \quad z \in \operatorname{int} \Gamma .
$$

Moreover,

$$
\frac{1}{2 \pi i} \int_{\Gamma} \frac{t f(t) \gamma(t)}{t-z} d t=f(z) u_{0}(z), \quad z \in \operatorname{int} \Gamma
$$

This is why

$$
\frac{1}{2 \pi i} \int_{\Gamma} \frac{t f(t)\left(\gamma(t)-v_{0}(t)\right)}{t-z} d t=0, \quad z \in \operatorname{int} \Gamma
$$

In the same way,

$$
\frac{1}{2 \pi i} \int_{\Gamma} \frac{g_{0}(t)\left(\gamma(t)-v_{0}(t)\right)}{t-z} d t=0, \quad z \in \operatorname{int} \Gamma
$$

For $z \in \operatorname{ext} \Gamma$ we let

$$
\alpha(z):=\frac{1}{2 \pi i} \int_{\Gamma} \frac{t f(t)\left(\gamma(t)-v_{0}(t)\right)}{t-z} d t, \quad \beta(z):=\frac{1}{2 \pi i} \int_{\Gamma} \frac{g_{0}(t)\left(\gamma(t)-v_{0}(t)\right)}{t-z} d t .
$$

Here the symbol ext $\Gamma$ stands for the exterior of $\Gamma$. It follows from the properties of Cauchy type integrals and its limiting values (see [1, Ch. I, Sect. 4]) that functions $\alpha$ and $\beta$ are analytic in ext $\Gamma$, can be analytically continued into some domain containing $\Gamma$, vanish at infinity, and

$$
t f(t)\left(\gamma(t)-v_{0}(t)\right)=\alpha(t) \text { and } g_{0}(t)\left(\gamma(t)-v_{0}(t)\right)=\beta(t) .
$$

for $t \in \Gamma$. We note that $\gamma(t)-v_{0}(t) \not \equiv 0$ on $\Gamma$. Indeed, otherwise function $v_{0}(t)$ can be analytically continued into the exterior of $\Gamma$ and it vanishes at infinity. Hence, it vanishes identically. At the same time, $\gamma \not \equiv 0$. It follows that

$$
\frac{f(t)}{g_{0}(t)}=\frac{\alpha(t)}{t \beta(t)}=: \frac{\alpha(t)}{\omega(t)}
$$

outside some finite set on $\Gamma$. This is why a meromorphic outside $\Gamma$ function $\alpha / \omega$ can be continued to a function $f / g_{0}$ meromorphic in $\Omega$. The obtained function meromorphic in $\mathbb{C}$ has finitely many poles and the infinity is either its zero or pole. It implies that $R:=\alpha / \omega$ is a rational function.

\section{BIBLIOGRAPHY}

1. F.D. Gakhov. Boundary value problems. Nauka, Moscow (1977). [Dover Publications New York (1990).]

2. Yu.A. Kaz'min. On successive error terms of Taylor series // Vestn. MGU. Ser. 1. Matem. Mekh. 5, 35-46 (1963). (in Russian).

3. N.E. Linchuk. Representation of commutants of the Pommiez operator and their applications // Matem. Zametki. 44:6, 794-802 (1988). [Math. Notes. 44:6, 926-930 (1988).]

4. N.I. Nagnibida. On a class of operators of generalized differentation in the space of functions analytic in a circle // Teor. Funkts. Funkts. Anal. Pril. Kharkov. 24, 98-106 (1975). (in Russian).

5. M.G. Khaplanov. On a completenetss of some system of analytic functions // Uchenye Zap. Rostov. Gos. Pedag. Univ. 3, 53-58 (1955). (in Russian).

6. Z. Binderman. Functional shifts induced by right invertible operators // Math. Nachr. 157:1, 211-224 (1992).

7. I.N. Dimovski, V.Z. Hristov. Commutants of the Pommiez operator // Int. J. Math. Math. Science. 2005:8, 1239-1251 (2005). 
8. G. Köthe. Dualität in der Funktionentheorie // J. Reine Angew. Math. 1953:191, 30-49 (1953).

9. Yu.S. Linchuk. Cyclical elements of operators which are left-inverses to multiplication by an independent variable // Meth. Funct. Anal. Topol. 12:4, 384-388 (2006).

10. M. Pommiez. Sur les zéros des reste successifs des séries de Taylor// Acad. Sci. Univ. Toulouse. 250:7, 1168-1170 (1960).

11. M. Pommiez. Sur les restes successifs des séries de Taylor // C.R. Acad. Sci. 250:15, 2669-2671 (1960).

12. M. Pommiez. Sur les restes et les dérivés des séries de Taylor // C.R. Acad. Sci. 251:17, 17071709 (1960).

13. M. Pommiez. Sur les différences divisées successives et les restes des séries de Newton généralisées // Ann. Fac. Sci. Univ. Toulouse. Ser. IV. 28, 101-110 (1964).

Ol'ga Alexandrovna Ivanova,

South Federal University,

I.I. Vorovich Institute of mathematics, mechanics and computer sciences,

Milchakova, 8a,

344090, Rostov-on-Don, Russia

E-mail: ivolga@sfedu.ru

Sergei Nikolaevich Melikhov,

Southern Federal University,

I.I. Vorovich Institute of mathematics, mechanics and computer sciences,

Milchakova str., 8a,

344090, Rostov-on-Don, Russia,

Southern Mathematical Institute, VSC RAS,

Markus str., 22,

362027, Vladikavkaz, Russia

E-mail: melih@math.rsu.ru 